\title{
Weniger Koanalgetika bei Krebspatienten nach Umstellung auf Methadon
}

Krebspatienten, die von anderen starken Opioiden auf Methadon umgestellt werden, benötigen danach weniger adjuvante Analgetika. Zudem scheint Methadon, einer japanischen Studie zufolge, neuropathische Schmerzen besser zu dämpfen als andere Opioidanalgetika.

E in krebsbedingter neuropathischer - Schmerz ist häufig therapieresistent und lässt die Lebensqualität der betroffenen Patienten erheblich sinken. Im Rahmen einer retrospektiven Studie hat das Wissenschaftlerteam um Yosuke Sugiyama von der japanischen Universitätsklinik in Nagoya nach neuen Behandlungsmöglichkeiten für neuropathische Schmerzen gesucht. Denn nahezu alle bislang verwendeten Koanalgetika machen die Patienten schläfrig und ihr vielfältiger Einsatz verschlechterte auf längere Sicht die Lebensqualität.

Eingeschlossen waren 28 stationäre Patienten, die bereits mit starken Opioiden wie Oxycodon oder Fentanyl sowie weiteren Schmerzmitteln durchschnittlich 321 Tage ohne ausreichenden Erfolg behandelt worden waren. 22 von ihnen litten auch oder nur unter neuropathischem Schmerz. Die Schmerzstärke wurde mit der FACES Pain Scale (FPS) auf einer Skala von 0 bis 5 und der neuropathische Schmerz nach dem Revised Edmonton Staging System und dem NeuPSIG Grading System bewertet. Per CT wurde die Nervenkompression durch den Tumor kontrolliert.

Die 24 innerhalb von zwei Wochen erfolgreich umgestellten Patienten waren von einer Methadondosis von anfangs durchschnittlich $18,8 \mathrm{mg} / \mathrm{d}$ auf $30,1 \mathrm{mg} / \mathrm{d}$ hochtitriert worden. Die durchschnittliche Dosis an Morphinäquivalenten einschließlich Methadon lag zu Beginn bei $156,2 \mathrm{mg} / \mathrm{d}$ und nach der Umstellung bei $169,2 \mathrm{mg} / \mathrm{d}$. Ob die Patienten nach der Umstellung noch ein adjuvantes Analgetikum erhielten oder nicht, beeinflusste die Methadondosis nicht signifikant $(25,8$ vs. $35,2 \mathrm{mg} / \mathrm{d})$. Das anfänglich feste tägliche Austauschverhältnis von Oxycodon: Methadon lag bei 5:1, Fentanyl $(\geq 100 \mu \mathrm{g} / \mathrm{h})$ : Methadon bei 20:1. Die ursprüngliche Opioiddosis wurde durch eine äquivalente Dosis Methadon ersetzt und schließlich beendet. Die äquivalente analgetische

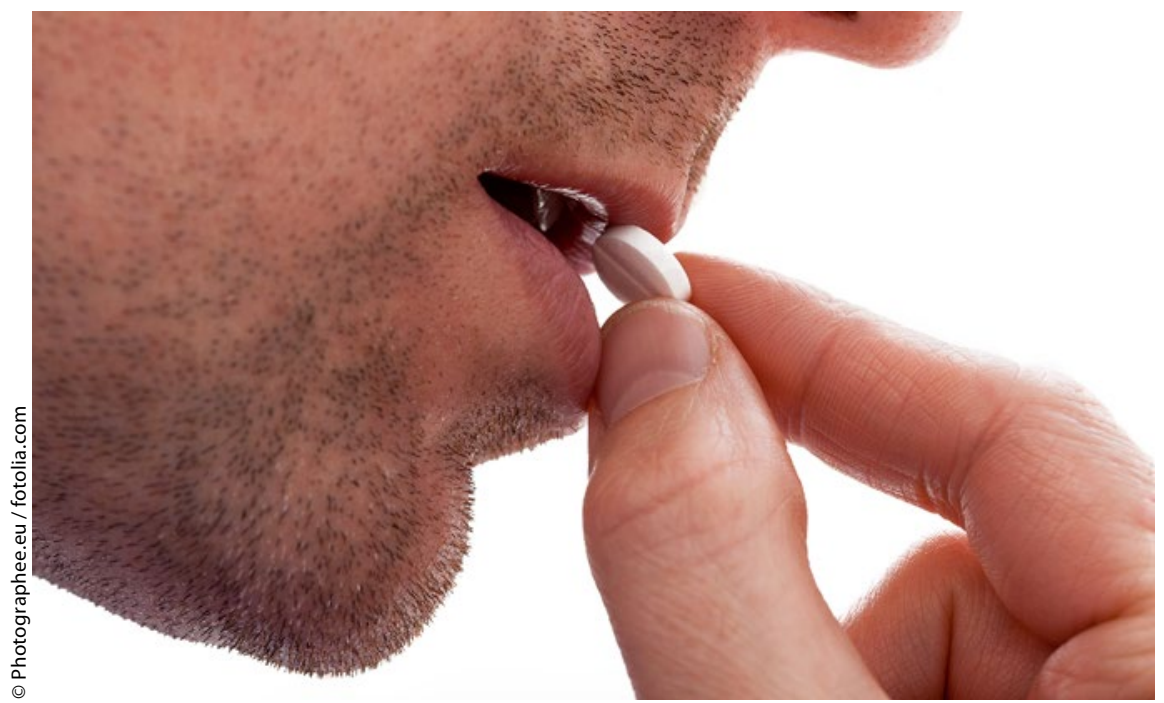

Ein Wechsel von starken Opioiden zu oralem Methadon kann Schmerzen bei Tumorpatienten verringern.
Dosierung wurde je nach Schmerzintensität und Stärke der Nebenwirkungen individuell angepasst.

85,7 \% der Patienten, die durch das palliativmedizinische Team auf Methadon umgesetzt wurden, erlebten innerhalb von 14 Tagen im FPS-Score eine durchschnittliche Schmerzreduktion von 4,43 auf 1,86. Zwei dieser Patienten verstarben zwei Wochen später an ihrem Krebsleiden. Die unter Methadon berichteten Arrhythmien waren in dieser Studie nicht zu beobachten. Wegen der Möglichkeit methadonbedingter verlängerter QT-Intervalle raten die Autoren dennoch, auf Komedikationen, Herzerkrankungen in der Vorgeschichte sowie eine vorbestehende Verlängerung der QT-Intervalle im EKG zu achten.

Bei 12 der 16 Patienten, die Koanalgetika wie Pregabalin, Kortikosteroide, Tramadol, Duloxetin und Neurotropin erhalten hatten, waren diese am Tag 14 nach der Umstellung auf Methadon nicht mehr oder nur noch in deutlich verringerter Anzahl erforderlich.

Fazit der Autoren: Die Ergebnisse der Studie legten nahe, so Sugiyama und Kollegen, dass die Umstellung von anderen starken Opioiden zu oralem Methadon nicht nur Schmerzen effektiver reduzieren könne, sondern auch die Möglichkeit eröffne, Koanalgetika deutlich einzuschränken. Dabei wirke Methadon sowohl über eine $\mu$-OpioidrezeptorStimulation als auch als N-Methyl-DAspartatrezeptor-Antagonist und Noradrenalin-Aufnahmehemmer. Dies verstärke die Wirkung von Methadon im Vergleich zu anderen Opioidanalgetika bei Krebspatienten mit neuropathischen Schmerzen, so Sugiyama und Kollegen. Die Umstellung verbessere somit die Lebensqualität nicht nur aufgrund der Schmerzreduktion, sondern auch durch eine Verringerung der Medikamentenzahl, verbunden mit weniger Nebenwirkungen.

Dr. Christine Starostzik

Sugiyama Y et al. A Retrospective Study on the Effectiveness of Switching to Oral Methadone for Relieving Severe Cancer-Related Neuropathic Pain and Limiting Adjuvant Analgesic Use in Japan. Journal of Palliative Medicine 2016; DOI: $10.1089 / j p m .2015 .0303$ 